\title{
Sez-6 May Play an Important Role in Neurite Outgrowth through the PKC $\gamma$ Signaling Pathways
}

\author{
Jing Zhang, ${ }^{\mathrm{a}, \#}$, Jun Zhang ${ }^{\mathrm{b}, \#, *}$, Youjun $\mathrm{Wu}^{\mathrm{a}}$, Yang Zhou ${ }^{\mathrm{b}}$, Zhihua Shao ${ }^{\mathrm{b}}$, \\ Xuan Kang ${ }^{\mathrm{b}}$, Lin $\mathrm{Ma}^{\mathrm{a}}$, Mengjie $\mathrm{Li}^{\mathrm{a}}$, Lihong Liu ${ }^{\mathrm{a}}$, and Hongjun Shi ${ }^{\mathrm{b}}$ \\ a Department of Regenerative Medicine, Tongji University, School of Medicine, Shanghai, \\ 200092, China. Fax:+86-21-65643250. E-mail: junzhang@tongji.edu.cn \\ b School of Life Science and Technology, Tongji University, Shanghai, 200092, China \\ * Author for correspondence and reprint requests \\ Z. Naturforsch. 66c, 614-620 (2011); received December 19, 2010/August 8, 2011
}

Seizure-related gene 6 (sez-6) was originally identified in a study of pentylenetetrazoletreated cortical neurons. Further studies on the structure and expression pattern suggested that Sez-6 may play an important role in neuronal development and function. In the present study, PC12 cells were used as a model to investigate the role of Sez-6 in neurite outgrowth. After a period of NGF treatment, the expression of Sez-6 in PC12 cells was increased. When Sez-6 expression was suppressed by the addition of an effective short hairpin RNA (shRNA) plasmid, the neurite outgrowth was significantly inhibited. In addition, we detected the expression level of protein kinase $\mathrm{C} \gamma(\mathrm{PKC} \gamma)$, and found that the $\mathrm{PKC} \gamma$ protein level was reduced in the differentiated PC12 cells but increased in PC12 cells lacking Sez-6. Taken together, our results indicate that Sez-6 acts on the neurite outgrowth of PC12 cells likely through the $\mathrm{PKC} \gamma$ signaling pathways.

Key words: Sez-6, PKC $\gamma$, Neurite Outgrowth

\section{Introduction}

Seizure-related gene 6 (sez-6) was originally isolated by differential hybridization from cortical neurons that were treated with pentylenetetrazole (PTZ), a convulsant drug known to induce epileptic seizures (Shimizu-Nishikawa et al., 1995). In response to PTZ, the expression of sez-6 was acutely up-regulated. Sez-6 has three isoforms, of which two, Sez-6 type I and type II, appear to be transmembrane proteins with long extracellular domains and short C-terminal cytoplasmic domains, while the type III isoform is a secreted protein identical to the amino terminal sequences of type I and type II except for $18 \mathrm{C}$ terminal amino acids (Kim et al., 2002). All Sez-6 isoforms contain the protein-protein interaction domains, short consensus repeats (SCRs) and complement subcomponent $\mathrm{C} 1 \mathrm{r}, \mathrm{C} 1 \mathrm{~s} / \mathrm{sea}$ urchin embryonic growth factor Uegf/bone morphogenetic protein 1 (CUB) (Gunnersen et al., 2007). Both domains are known to be involved in signaling, cell-cell adhesion, and recognition (Yu et al., 2007). This structure suggests that Sez-6 would be involved in membrane receptor signaling.

\footnotetext{
\# These authors contributed equally to this work.
}

Sez-6 is expressed in the brain and testis in addition to other neural tissues. In the brain, sez-6 mRNA expression is prominent in the neurons of the hippocampus, olfactory tubule, and certain sublayers of the neocortex (Herbst and Nicklin, 1997). Researchers have noted a highly restricted stage-specific expression of sez-6 mRNA in the mouse forebrain development (Kim et al., 2002). A recent study on Sez-6 null mutant mice has revealed a role for Sez-6 in dentritic patterning and synaptic regulation (Gunnersen et al., 2007). Taken together, Sez-6 may play an important role in neuronal development and function.

Protein kinase $\mathrm{C} \gamma(\mathrm{PKC} \gamma)$ is a member of the classical protein kinase $\mathrm{C}$ (PKC) subfamily that is selectively expressed in most neurons including cerebellar Purkinje cells and hippocampal pyramidal neurons (Saito and Shirai, 2002). Subcellular localization studies indicated that $\mathrm{PKC} \gamma$ is present primarily in the soma and dendritic processes of neurons (Kano et al., 1995). Previous studies have shown that PKC $\gamma$ may play an important role in the dendritic development of Purkinje cells. In PKC $\gamma$ deficient mice, Purkinje cell dendritic trees were enlarged with an increased number of branching points compared to wild-type mice. Pharma- 
cological $\mathrm{PKC} \gamma$ activation by the phorbol ester phorbol-12-myristate-13-acetate (PMA) induced a retraction of dendritic branches whereas $\mathrm{PKC} \gamma$ inhibition by GF109203X promoted the branching of higher-order dendrites (Schrenk et al., 2002). These results suggest that the $\mathrm{PKC} \gamma$ isoform is a negative regulator of dendritic growth and branching.

The rat pheochromocytoma cell line PC12 is a well-established model system used extensively for studying mechanisms involved in neuronal differentiation (Greene and Tischler, 1976). In response to nerve growth factor (NGF), PC12 cells cease division and differentiate into sympathetic neuron-like cells with extensive neurites (Greene et al., 1987). In the present study, we designed two short hairpin RNA (shRNA) plasmids targeting the rat Sez-6 to investigate Sez-6's role in the differentiation of PC12 cells. In the absence of Sez-6, the neurite outgrowth of PC12 cells was markedly inhibited, and the $\mathrm{PKC} \gamma$ protein expression in these cells was significantly increased. This study indicates that Sez-6 is an essential component in the neurite outgrowth of PC12 cells and it may act through the $\mathrm{PKC} \gamma$ signaling pathways.

\section{Material and Methods}

\section{shRNA plasmids construction}

According to the shRNA design principle (Tuschl, 2002), two 19-bp sequences targeting the rat Sez-6 (GenBank: XM_239260) were selected. One was 5'-GACCACAGGGCTTGGTATC-3' (bp 654-672) and the other was 5'-GAGCCTGTCTGCATTGCTG-3' (bp 1267-1285). The loop sequence (TTCAAGAGA) chosen for the shRNA was suggested by Brummelkamp et al. (2002). The deoxyoligo nucleotides containing the shRNA target sequence, the loop sequence, the reverse complement of the target sequence, an RNA polymerase III termination sequence (TTTTT), and restriction-site compatible overhangs were designed and purchased from Sangon Biotech (Shanghai, China). Double complementary DNA segments were gained through annealing, named Sez-6-1shRNA and Sez-6-2-shRNA, and then inserted into the pSUPER-EGFP1 plasmid. The recombinant plasmids were identified by DNA sequence analysis.

\section{Cell culture and transfection}

PC12 cells were purchased from Cell Bank of Chinese Academy of Sciences (Shanghai, China) and cultured routinely in Dulbecco's modified Eagle's medium (DMEM) supplemented with $5 \%$ fetal bovine serum (FBS) (Gibco, Carlsbad, CA, USA) and $10 \%$ heat-inactivated horse serum (Gibco). To induce differentiation, cells were exposed to $50 \mathrm{ng} / \mathrm{mL} 2.5 \mathrm{~S}$ NGF (Sigma, St. Louis, MO, USA) in DMEM supplemented with $1 \%$ FBS and $2 \%$ horse serum for the indicated time. NGF-treated PC12 cells were transiently transfected with the shRNA expression plasmid Sez6-1-shRNA or Sez-6-2-shRNA using Lipofectami$\mathrm{ne}^{\mathrm{TM}} 2000$ (Invitrogen, Carlsbad, CA, USA) according to the manufacturer's instructions. Untransfected cells and cells transfected with empty plasmid served as control groups. Forty eight h after transfection, cells were harvested and analysed by real time reverse transcription-polymerase chain reaction (RT-PCR), Western blotting, and immunocytochemistry.

\section{Real time RT-PCR}

Total RNA was extracted using Trizol (Invitrogen) and reverse-transcribed using Superscript ${ }^{\mathrm{TM}}$ II (Invitrogen). The sense and antisense primers for Sez-6 were 5'-ACTTCTTTGAGGTGCCCCGCAATG-3' and 5'-CCGCTTGACGATCATGGCAAGTAAG-3' (357 bp). The primers for $\beta$-actin, which served as an internal control, were 5'-GCCACTGCCGCATCCTCTT-3' and 5'-GGCATCGGAACCGCTCA-3' (91 bp). SYBR Green quantitative PCR amplifications were performed on the Rotor-Gene 3000 real-time PCR system (Qiagen, Hilden, Germany). Reactions were carried out in a volume of $25 \mu \mathrm{L}$ containing $12.5 \mu \mathrm{L}$ of $2 \times$ SYBR Premix Ex Taq ${ }^{\mathrm{TM}}$ (TaKaRa Biotechnology Co., Ltd., Dalian, China). The comparative $\mathrm{Ct}\left(2^{-\Delta \Delta \mathrm{Ct}}\right)$ method was used for analysis. Each sample was assayed in 3 replicates in at least two separate experiments.

\section{Production of anti-Sez-6 antiserum}

Antiserum production was conducted according to the standard protocols. A peptide corresponding to a portion of the rat Sez- 6 protein sequence (74EEFLQEGLEGREEAP88) was synthesized by TaKaRa Biotechnology Co., Ltd. and conjugated to keyhole limpet hemocyanin $(\mathrm{KLH})$. The conjugate KLH-peptide was emulsified in Freund's complete adjuvant (Sigma, Natick, MA, USA) and injected at multiple subcutaneous sites into the New Zealand white 
rabbits. Boost injections of the conjugate emulsified in Freund's incomplete adjuvant were given at 2-week-intervals. Blood was collected before each immunization, and the serum antibody titer was determined by indirect ELISA using the peptide antigen. When the titer was above 1:10000, rabbits were bled 1 week after the final injection. Sera were separated from blood and stored in aliquots at $-80^{\circ} \mathrm{C}$.

\section{Western blot analysis}

To determine the expression level of Sez-6 and PKC $\gamma$, total cellular proteins were extracted using RIPA lysis buffer (Santa Cruz, Santa Cruz, CA, USA), and the concentration was determined by the Bio-Rad Bradford protein assay method (Bio-Rad, Hercules, CA, USA). Equal amounts of protein were separated by $8 \%$ sodium dodecyl sulfate (SDS)-polyacrylamide gel electrophoresis and transferred to polyvinylidene difluoride membranes (Millipore, Billerica, MA, USA). The membranes were blocked with $5 \%$ non-fat dry milk dissolved in Tris-buffered saline with $0.1 \%$ Tween-20 (TBST) and then incubated overnight at $4{ }^{\circ} \mathrm{C}$ with rabbit anti-Sez-6 antiserum (1:400) or mouse anti- $\beta$-actin IgG mAb (1:4000; Sigma) or rabbit anti-PKC $\gamma \operatorname{IgG}$ pAb (1:500; Santa Cruz). After being washed with TBST, the membranes were incubated with horseradish peroxidase (HRP)-conjugated goat anti-rabbit IgG (1:4000; Santa Cruz) or HRP-conjugated goat anti-mouse IgG (1:4000; Santa Cruz) for $1 \mathrm{~h}$ at room temperature. Bands were visualized by the enhanced chemiluminescence technique and quantified by densitometry. $\beta$-Actin was used for normalization.

\section{Immunocytochemistry}

NGF-treated PC12 cells were grown on poly-Llysine-coated coverslips. Forty eight $\mathrm{h}$ after transfection, cells were fixed in $4 \%$ paraformaldehyde (PFA) and permeabilized with $0.1 \%$ Triton X-100 in phosphate-buffered saline (PBS). After being blocked with $5 \%$ normal goat serum and $0.1 \%$ Triton X-100 in PBS, cells were incubated at $4{ }^{\circ} \mathrm{C}$ overnight with rabbit anti-Sez-6 antiserum (1:50). Coverslips were rinsed in PBS, incubated in the dark with rhodamine-conjugated goat anti-rabbit secondary antibody (1:200; Millipore) for $2 \mathrm{~h}$ at room temperature, then rinsed again in PBS, and mounted with Vectashield mounting medium, sealed with nail polish, and viewed using a laserscanning confocal microscope.

\section{Measurement of neurite length}

To measure the neurite length, images were captured using a laserscanning confocal microscope. For each treatment, a total of 300 cells (100 cells per independent culture) were selected to analyse. The total neurite length was measured by the Motic Images Advanced 3.2 Software (Motic China Group Co., Ltd., Shanghai, China), and the average neurite length was calculated.

\section{Statistical analysis}

Data were expressed as mean \pm SD. Statistical analyses were conducted with Student's t test and performed with SPSS 10.0 software. $P<0.05$ was considered statistically significant.

\section{Results}

\section{Effect of Sez-6 shRNA on Sez-6 expression}

In order to determine the role of Sez- 6 in the differentiation of $\mathrm{PC} 12$ cells, we designed two plasmids, Sez-6-1-shRNA and Sez-6-2-shRNA, to express shRNA targeting rat Sez-6, and transfected NGF-treated PC12 cells with these two shRNA plasmids as well as empty plasmid as control. Forty eight hours later, Sez-6 mRNA and protein expression levels in the cells were estimated.

The effects of Sez-6 shRNA on mRNA and protein expression were evaluated by real time RT-PCR and Western blotting separately, and the results are displayed in Fig. 1. After treatment with NGF for several days, Sez-6 expression in the differentiated PC12 cells increased significantly as compared with the pre-differentiated cells $(P<0.05)$. As expected, Sez-6 expression in the untransfected NGF-treated cells and the control empty plasmid transfected cells were similar $(P>0.05)$. The statistical analysis also showed that Sez-6 mRNA and protein levels of the Sez6-1-shRNA transfected cells were reduced compared with that of control PC12 cells without NGF and transfection $(P<0.05)$ and the inhibition rate reached approximately $50 \%$. However, no difference was seen between the Sez-6-2-shRNA transfected cells and the control $(P>0.05)$. These results suggested that only Sez-6-1-shRNA was the effective shRNA to suppress the expression of Sez-6. 
(A)

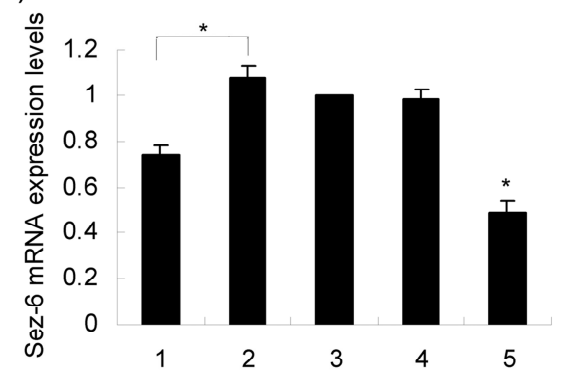

(B)

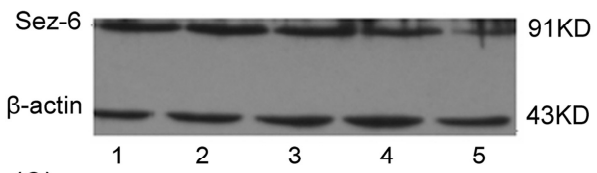

(C)

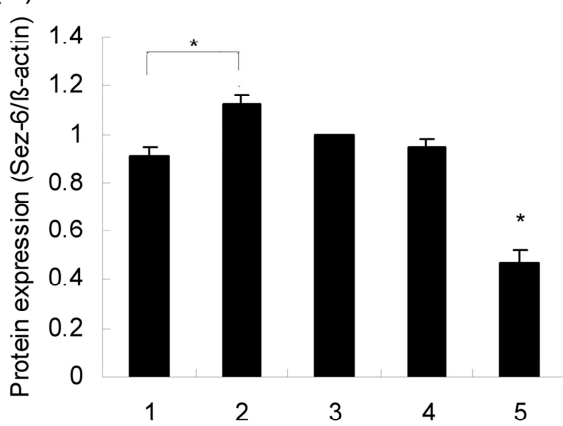

Fig. 1. The effect of Sez-6 shRNA on Sez-6 expression. (A) Sez-6 mRNA expression levels detected by real time RT-PCR. (B) Western blots of Sez-6 protein levels. (C) Quantitative expression of Sez-6 protein levels. 1, PC12 cells - NGF; 2, PC12 cells + NGF; 3, PC12 cells + NGF and empty plasmid for 48 h; 4, PC12 cells + NGF and Sez-6-2-shRNA for 48 h; 5, PC12 cells + NGF and Sez-6-1-shRNA for $48 \mathrm{~h}$. Bands corresponding to Sez-6 and $\beta$-actin protein were scanned and the density was determined by optical density measurement. Results are shown as the mean \pm SD. $* P<0.05$.
(A)

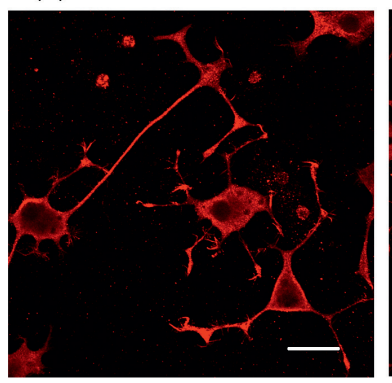

(B)

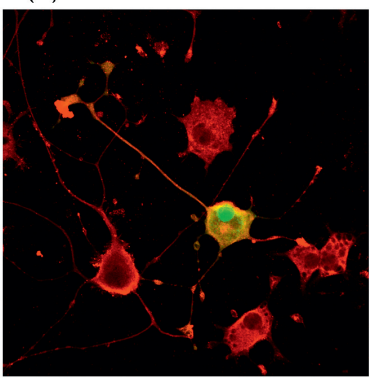

(A)

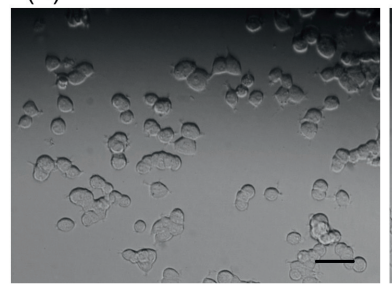

(C)

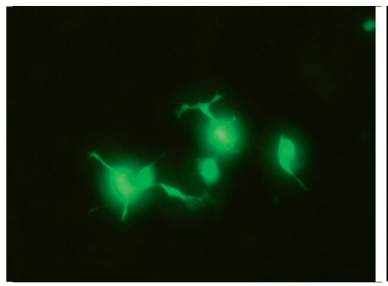

(B)

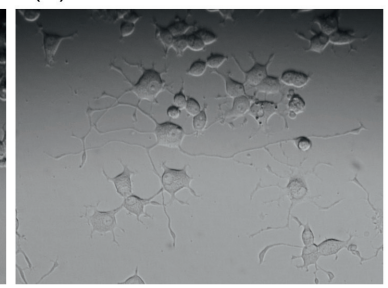

(D)

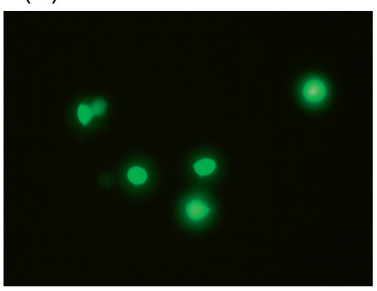

Fig. 2. Morphological characteristics of PC12 cells. (A) In the absence of NGF. (B) PC12 cells cultured in $50 \mathrm{ng} /$ mL NGF. (C) NGF-treated PC12 cells transfected with empty plasmid for $48 \mathrm{~h}$ under a fluorescent microscope. (D) NGF-treated PC12 cells transfected with Sez-6-1shRNA for $48 \mathrm{~h}$ under a fluorescent microscope. Scale bar, $250 \mu \mathrm{m}$.

\section{Effect of Sez-6 shRNA on neurite outgrowth of PC12 cells}

In the absence of NGF, PC12 cells were round to ovoid in shape, with few neurites (Fig. 2A). After several days of exposure to NGF, the cells ceased to multiply and initiated neurite outgrowth similar to sympathetic neurons (Fig. 2B). In order to study the role of Sez-6 in neurite outgrowth, we transfected NGF-treated PC12 cells with empty plasmid or Sez-6-1-shRNA, and observed EGFP fluorescence and cellular morphological changes under a fluorescence microscope $48 \mathrm{~h}$ post trans-
(C)

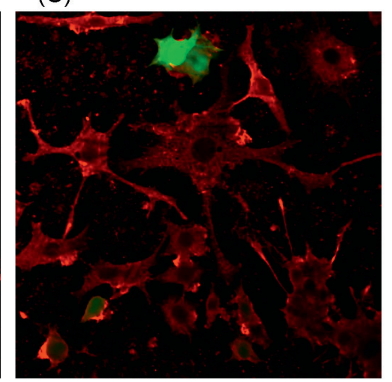

Fig. 3. Localization of Sez-6 in PC12 cells. (A) PC12 cells cultured in 50 ng/mL NGF. (B) NGF-treated PC12 cells transfected with empty plasmid for $48 \mathrm{~h}$. (C) NGF-treated PC12 cells transfected with Sez-6-1-shRNA for $48 \mathrm{~h}$. Immunoreactivity of Sez-6 was visualized with rhodamine-conjugated secondary antibody (red) and co-localization of EGFP fluorescence (green) was observed using a confocal laser scanning microscope. Scale bar, $50 \mu \mathrm{m}$. 
(A)
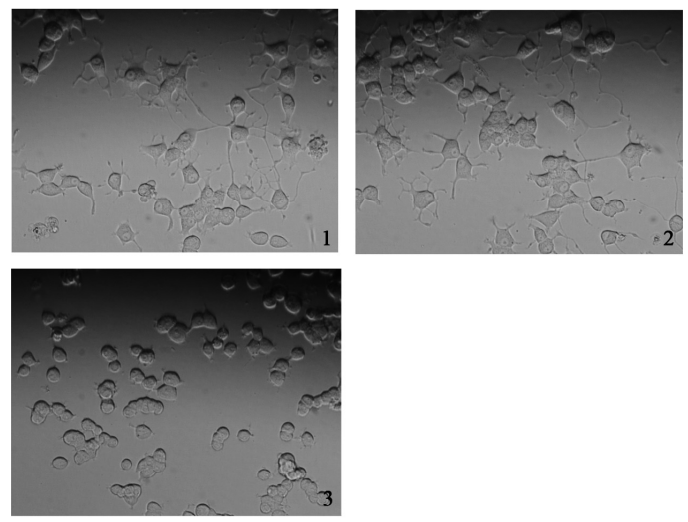

(B)

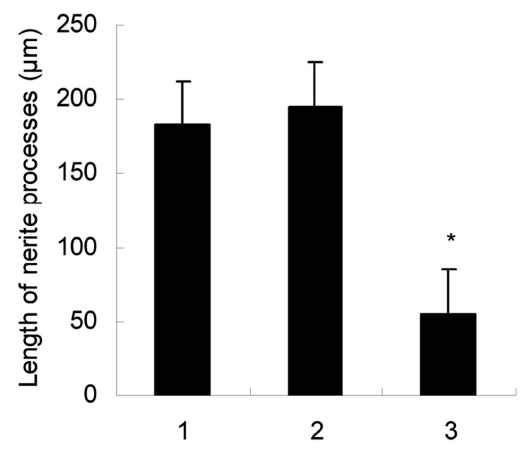

Fig. 4. Measurement of the mean neurite length of NGF-treated PC12 cells. (A) Phase-contrast images were captured by a digital CCD camera equipped in an inverted microscope at 100x. (B) $48 \mathrm{~h}$ after transfection, neurite length of cells was analysed by camera software. Data are means \pm S. E. $(n=3)$. 1 , PC12 cells cultured in $50 \mathrm{ng} / \mathrm{mL}$ NGF; 2, NGF-treated PC12 cells transfected with empty plasmid for $48 \mathrm{~h} ; 3$, NGF-treated PC12 cells transfected with Sez-6-1-shRNA for $48 \mathrm{~h}$. Results are shown as the mean $\pm \mathrm{SD}$. $* P<0.05$.

fection. As shown in Fig. 2C, the cells treated with empty plasmid developed long neurites similar to the untransfected cells. In contrast, Sez-6-1shRNA transfected cells showed a significant inhibition of neurite outgrowth (Fig. 2D).

\section{Localization of Sez-6 in PC12 cells treated with Sez-6 shRNA}

Immunofluorescence observed under a confocal microscope indicated the location of Sez-6 protein. As shown in Fig. 3, the red fluorescence representing Sez-6 protein was located around the cell membrane. Intrinsic EGFP fluorescence

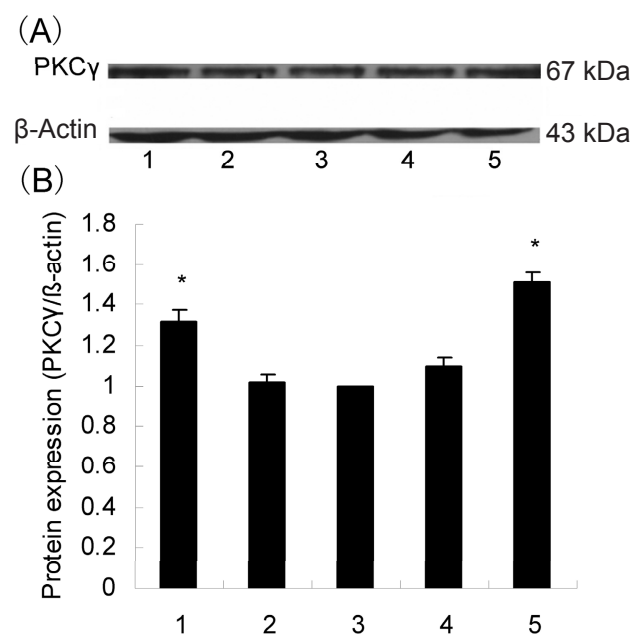

Fig. 5. PKC $\gamma$ protein expression detected by Western blotting. (A) Western blots of PKC $\gamma$ protein levels. (B) Quantitative expression of PKC $\gamma$ levels. 1, PC12 cells NGF; 2, PC12 cells + NGF; 3, PC12 cells + NGF and empty plasmid for $48 \mathrm{~h} ; 4$, PC12 cells + NGF and Sez6-2-shRNA for $48 \mathrm{~h} ; 5, \mathrm{PC} 12$ cells + NGF and Sez-61-shRNA for $48 \mathrm{~h}$. Bands corresponding to $\mathrm{PKC} \gamma$ and $\beta$-actin protein were scanned and the density was determined by optical density measurement. Results are shown as the mean $\pm \mathrm{SD}$. $* P<0.05$.

was green. After transfection with the empty plasmid, the red and green fluorescence merged and turned yellow. In the Sez-6-1-shRNA transfected cells, the red fluorescence was not as visible as in the untransfected cells, but showed visible green fluorescence. The results confirmed the effect of Sez-6-1-shRNA on down-regulation of Sez-6 expression.

From the images, we found that the neurite length of Sez-6-1-shRNA transfected cells was shorter than those of the surrounding untransfected cells and the empty plasmid transfected cells. Further, the length of neurite processes was measured and compared to controls (Fig. 4). The statistical analysis showed that there was no significant difference in the neurite length between the untransfected cells and the controls $(P>$ $0.05)$. However, the neurite length of the Sez-6-1shRNA transfected cells was reduced by $71.87 \%$ compared with the control $(P<0.05)$.

$P K C \gamma$ expression in PC12 cells treated with Sez-6 shRNA

$\mathrm{PKC} \gamma$ is a neuron-specific PKC isoform. It has already been proved that $\mathrm{PKC} \gamma$ is a negative regu- 
lator of dendritic growth and branching. To determine whether the inhibition of neurite outgrowth induced by the reduction in Sez-6 expression is related to PKC $\gamma$, the Western blotting method was used (Fig. 5). Consistent with previous studies, the expression of $\mathrm{PKC} \gamma$ protein in the differentiated PC12 cells was reduced significantly compared with the pre-differentiated cells $(P<0.05)$. After transfected with Sez-6-1-shRNA, we found that the $\mathrm{PKC} \gamma$ protein expression in these cells was increased approximately by $50 \%$ compared with the control $(P<0.05)$. The cells transfected with Sez-6-2-shRNA, which had no significant effect on down-regulation of Sez-6 expression as already confirmed above, $\mathrm{PKC} \gamma$ protein equivalent expressed to the control $(P>0.05)$. These results suggested that the role of Sez-6 in neurite outgrowth may be correlated with $\mathrm{PKC} \gamma$ protein.

\section{Discussion}

RNA interference (RNAi) is the process of sequence-specific post-transcriptional gene silencing by the introduction of homologous double-stranded RNA (dsRNA) into the cells (Hammond et al., 2000; Dillon et al., 2005). Two kinds of dsRNA, small interfering RNA (siRNA) (Elbashir et al., 2001) and vector-mediated expression short hairpin RNA (shRNA) (Paddison et al., 2002; Sui et al., 2002), are mainly used to suppress gene expression in gene function studies. The ability of shRNA to silence gene expression can be evaluated by real time RT-PCR, Western blot analysis, and immunocytochemistry. In this study, we designed two plasmids, Sez-6-1-shRNA and Sez-6-2-shRNA, to express shRNA targeting rat Sez-6 and introduced them into the NGF-treated PC12 cells. In the Sez-6-1-shRNA transfected cells, the Sez- 6 mRNA and protein expression levels were both reduced by approximately $50 \%$ compared to the controls. The further immunocytochemistry results confirmed the effect of Sez6-1-shRNA on down-regulation of Sez-6 expression. However, no difference was seen between the Sez-6-2-shRNA transfected cells and the controls. This means that Sez-6-2-shRNA failed to suppress Sez-6 expression, although this vector could yield a homologous transcript targeting Sez-6. These results demonstrate that gene silencing by shRNA is highly target sequence-specific.

Sez-6 is the only cDNA in the nervous system that encodes both SCR and CUB domains, which suggests that Sez-6 may play a role in neuronal membrane signaling (Yu et al., 2007). In a recent study on Sez-6 null mutant mice, researchers found that pyramidal cortical neurons exhibited increased dendritic branching, but a decrease in mean neurite length. The knockout neurons showed reduced excitatory postsynaptic responses and significantly fewer excitatory synapses (Gunnersen et al., 2007). In our study, we used NGF-treated PC12 cells as a model to investigate Sez-6's role in neurite outgrowth. Sez-6 expression can be detected in these PC12 cells before and after NGF treatment, and after treatment the expression level was raised. After transfected with the shRNA plasmids, the PC12 cells lacking Sez-6 showed an inhibition in neurite outgrowth. Under the confocal microscope, a significantly decreased neurite length was observed. These results confirm that Sez-6 plays an important role in neurite development.

Neurite outgrowth is a crucial step in neural development. In recent years, a number of molecules have been reported to regulate neurite outgrowth. For example, neurotrophin 3 (NT-3), brain-derived neurotrophic factor (BDNF), nerve growth factor (NGF), insulin-like growth factor I, and bone morphogenetic protein 7 can act as extrinsic factors to influence the dendritic morphology of neurons (Schrenk et al., 2002; McAllister et al., 1995; Withers et al., 2000; Niblock et al., 2000; Jan and Jan, 2003). In addition to extrinsic regulators, many intracellular molecules such as small GTPases like Rho, Rac, and cdc42 have been shown to be important for dendritic development (Jan and Jan, 2003; Threadgill et al., 1997). Researchers have also found that many of these pathways interact with PKC signaling (Metzger and Kapfhammer, 2000, 2003). PKC $\gamma$ is a multifunctional signaling molecule activated by $\mathrm{Ca}^{2+}$ and diacylglycerol. Upon activation, PKC phosphorylates a number of proteins associated with neuronal development (Metzger and Kapfhammer, 2003). Previous studies have already confirmed that $\mathrm{PKC} \gamma$ is a negative regulator of dendritic growth (Schrenk et al., 2002). In our study, we detected $\mathrm{PKC} \gamma$ protein expression in $\mathrm{PC} 12$ cells, and found that the $\mathrm{PKC} \gamma$ protein level was reduced in the differentiated PC12 cells but increased in PC12 cells lacking Sez-6. These results suggest that Sez-6 in neurite outgrowth may act through the $\mathrm{PKC} \gamma$ signaling pathways. How- 
ever, the precise molecular mechanisms of Sez-6's function on neurite outgrowth are not yet known.

NGF-induced PC12 cells are a well-established model system for neurite outgrowth. In this study, we have successfully constructed a shRNA plasmid which suppresses Sez-6 expression. Further experiments indicated that Sez-6 affects the elongation of neurites of PC12 cells and likely acts through the $\mathrm{PKC} \gamma$ signaling pathways. Thus, through studying the role of Sez-6 on neurite outgrowth, it is helpful to further understand the

Brummelkamp T. R., Bernards R., and Agami R. (2002), A system for stable expression of short interfering RNAs in mammalian cells. Science 296, 550-553.

Dillon C. P., Sandy P., Nencioni A., Kissler S., Rubinson D. A., and Van Parijs L. (2005), RNAi as an experimental and therapeutic tool to study and regulate physiological and disease processes. Annu. Rev. Physiol. 67, 147-173.

Elbashir S. M., Harborth J., Lendeckel W., Yalcin A., Weber K., and Tuschl T. (2001), Duplexes of 21-nucleotide RNAs mediate RNA interference in cultured mammalian cells. Nature 411, 494-498.

Greene L. A. and Tischler A. S. (1976), Establishment of a noradrenergic clonal line of rat adrenal pheochromocytoma cells which respond to nerve growth factor. Proc. Natl. Acad. Sci. USA 73, 2424-2428.

Greene L. A., Aletta J. M., Rukenstein A., and Green S. H. (1987), PC12 pheochromocytoma cells: culture, nerve growth factor treatment, and experimental exploitation. Methods Enzymol. 147, 207-216.

Gunnersen J. M., Kim M. H., Fuller S. J., De Silva M., Britto J. M., Hammond V. E., Davies P. J., Petrou S., Faber E. S., Sah P., and Tan S. S. (2007), Sez-6 proteins affect dendritic arborization patterns and excitability of cortical pyramidal neurons. Neuron 56, 621-639.

Hammond S. M., Bernstein E., Beach D., and Hannon G. J. (2000), An RNA-directed nuclease mediates post-transcriptional gene silencing in Drosophila cells. Nature 404, 293-296.

Herbst R. and Nicklin M. J. (1997), SEZ-6: promoter selectivity, genomic structure and localized expression in the brain. Brain Res. Mol. Brain Res. 44, 309-322.

Jan Y. N. and Jan L. Y. (2003), The control of dendrite development. Neuron 40, 229-242.

Kano M., Hashimoto K., Chen C., Abeliovich A., Aiba A., Kurihara H., Watanabe M., Inoue Y., and Tonegawa S. (1995), Impaired synapse elimination during cerebellar development in PKC gamma mutant mice. Cell 83, 1223-1231.

Kim M. H., Gunnersen J. M., and Tan S. S. (2002), Localized expression of the seizure-related gene SEZ-6 in developing and adult forebrains. Mech. Dev. 118, $171-174$.

McAllister A. K., Lo D. C., and Katz L. C. (1995), Neurotrophins regulate dendritic growth in developing visual cortex. Neuron 15, 791-803. mechanisms of neuronal function and dysfunction.

\section{Acknowledgements}

This research was supported by Natural Science Foundation of China (30570690 and 81041069), International Cooperation Program of the Ministry of Science and Technology of China (2011DFA30480), and National Key Basic Research Program of China (2011CB965102).

Metzger F. and Kapfhammer J. P. (2000), Protein kinase $\mathrm{C}$ activity modulates dendritic differentiation of rat Purkinje cells in cerebellar slice cultures. Eur. J. Neurosci. 12, 1993-2005.

Metzger F. and Kapfhammer J. P. (2003), Protein kinase $\mathrm{C}$ : its role in activity-dependent Purkinje cell dendritic development and plasticity. Cerebellum 2, 206-214.

Niblock M. M., Brunso-Bechtold J. K., and Riddle D. R. $(200 \bullet)$, Insulin-like growth factor I stimulates dendritic growth in primary somatosensory cortex. J. Neurosci. 20, $4165-4176$.

Paddison P. J., Caudy A. A., Bernstein E., Hannon G. J., and Conklin D. S. (2002), Short hairpin RNAs (shRNAs) induce sequence-specific silencing in mammalian cells. Genes Dev. 16, 948-958.

Saito N. and Shirai Y. (2002), Protein kinase C gamma (PKC gamma): function of neuron specific isotype. J. Biochem. 132, 683-687.

Schrenk K., Kapfhammer J. P., and Metzger F. (2002), Altered dendritic development of cerebellar Purkinje cells in slice cultures from protein kinase $\mathrm{C}$ gamma-deficient mice. Neuroscience 110, 675-689.

Shimizu-Nishikawa K., Kajiwara K., Kimura M., Katsuki M., and Sugaya E. (1995), Cloning and expression of SEZ-6, a brain-specific and seizure-related cDNA. Brain Res. Mol. Brain Res. 28, 201-210.

Sui G., Soohoo C., Affar el B., Gay F., Shi Y., and Forrester W. C. (2002), A DNA vector-based RNAi technology to suppress gene expression in mammalian cells. Proc. Natl. Acad. Sci. USA 99, 5515-5520.

Threadgill R., Bobb K., and Ghosh A. (1997), Regulation of dendritic growth and remodeling by Rho, Rac, and Cdc42. Neuron 19, 625-634.

Tuschl T. (2002), Expanding small RNA interference. Nat. Biotechnol. 20, 446-448.

Withers G. S., Higgins D., Charette M., and Banker G. (2000), Bone morphogenetic protein-7 enhances dendritic growth and receptivity to innervation in cultured hippocampal neurons. Eur. J. Neurosci. 12, $106-116$.

Yu Z. L., Jiang J. M., Wu D. H., Xie H. J., Jiang J. J., Zhou L., Peng L., and Bao G. S. (2007), Febrile seizures are associated with mutation of seizure-related (SEZ) 6, a brain-specific gene. J. Neurosci. Res. 85, 166-172. 